\title{
Prediction of Water-Soluble Polymer Drag Reduction Performance in Multiphase Flow Using Fuzzy Logic Technique
}

\author{
M. Eshrati*, A. R. Al-Hashmi, M. Ranjbaran, T. Al-Wahaibi, Y. Al-Wahaibi, A. Al-Ajmi \\ and A. Abubakar
}

\begin{abstract}
An experimental study on the turbulent drag reduction in dispersed oil-water flow using different polymers has been conducted at different polymer concentrations and different flow conditions. This study presents the use of fuzzy logic for investigation of drag reduction performance of water-soluble polymers in multiphase flow in pipeline, operated with tap water and model oil. Fuzzy logic is more useful in connecting the multiple inputs to a single output and in contrast to the traditional logics. The objective of this study is to investigate the relationship between drag reduction and working parameters such as molecular weight, mixture velocity, charge density, polymer concentration and oil fraction, to determine how fuzzy logic performs in prediction of drag reduction (DR\%). For this purpose several anionic polymers were applied in a two phase flow through a horizontal plexiglass pipe flow loop system of 30.6-mm ID. A 1000 ppm master solutions of each polymer were injected at different mixture flow rates to achieve polymer concentrations in the range of 5 to $30 \mathrm{ppm}$ (optimum concentration) in the mixture flowing through the test section. The drag reduction increased with polymer concentration up to $10 \mathrm{ppm}$, above which it reached a plateau value. The results showed that drag reduction increases with polymer molecular weight and mixture velocity increase, and the maximum drag reduction affected by the increase in polymer charge density and oil fraction. The minimum drag reduction observed (at oil fraction of 0.5 ) around $5 \%$ and the maximum (always at 0.1 oil fraction) around $60 \%$. Based on the results a fuzzy logic model has developed for the prediction of drag reduction and the prediction is also verified through various results of the rest of polymer. In addition a dimensional analysis is performed to find the best fitting equation to the results, and result shows that a quadratic form of the proposed equation is the most appropriate one among other possible form of equations.
\end{abstract}

Keywords-Multiphase oil-water flow, drag reduction, Fuzzy Logic, molecular weight, charge density, mixture velocity, Dimensional Analysis.

\section{INTRODUCTION}

Since Toms discovery of drag reduction in 1948 [1], extensive studies on drag reducing polymers (DRPs) has been done ([2], [3], [4], [5]). The successful implementation of this technology has been accomplished in the Trans-Alaska pipeline[6]. DRPs' various specifications such as chemical

\footnotetext{
Department of Petroleum and Chemical Engineering, Sultan Qaboos University,

*Corresponding author at: Department of Petroleum and Chemical Engineering,
}

structure, molecular weight and intrinsic viscosity were investigated at different fluid velocity and pipe diameters ([7], [8], [9], [10], [11], [12]). Drag reduction in multiphase flow is not so straight forward as single phase due to the occurrence of different flow patterns at different oil/water ratios and mixture velocities. However, drag reduction phenomenon is not well understood, and a number of aspects of drag reduction remain to be investigated. In general, the previous studies indicate that any polymer of an adequately high molecular weight (greater than $1 \times 105)$, or any one which can form high molecular weight aggregates, will performs as an efficient DRP ([13], [14], [15]). The effectiveness of drag reduction is dependent on several factors, such as polymer molecule size and its conformation, oil properties, molecular interactions, flow rate, polymer concentration, turbulent intensity and flow geometry, [16].

However some correlations in single phase were developed employing a polymer/oil specific model that expresses the drag reduction in terms of DRA concentration, [6],

$$
\frac{1}{D R}=\frac{A}{p p m}+B
$$

But a generic model in the multiphase flow has not received attention, in which the polymeric parameter and flow geometry are the limiting factors. In the series of preliminary tests these factors have significant effect on drag reduction, (such as density, flowrate, oil fraction, viscosity and pipe diameter), although experiments to study all individual factors were robust but by means of dimensional analysis a new equation for DR prediction in the multiphase system applied, and between different forms of possible equation the most appropriate one is picked. Fuzzy logic imitates human reasoning, calculations and operations via fuzzy groups and linguistic variables, expressions such as very low, low, high, very high and so on, are used in fuzzy logic as the linguistic variables.

The concept of fuzzy logic is notably suitable for processes with high levels of complexity and uncertainty, oil and gas industry since its inception encountered these types of processes. Hence many processes rely on the expertise and personnel's skills, the fuzzy logic provides personnel to incorporate their knowledge of experienced operations into the control of these processes. A successful example is the enhancement of operation of an engine unit of a pumping station, [17], and or the design facilitation of a control system as a control support, by application of fuzzy inference to the 
digital control of pipelines, [18].

Efforts towards relating measurable properties to DRA performance in both single and multiphase flow have been undertaken since at least the 1960s. For the purpose of DR prediction a new algorithm was developed using fuzzy logic technique to correlate the considered parameters such as molecular weight, charge density, polymer concentration, and oil fraction and mixture velocity. Hence working with five manipulating parameter is robust the Fuzzy modeling of systems which is a technique for the treatment of qualitative information in a rigorous way is chosen, where allowing the reduction of the project complexity, [19], [20].

On the other side a dimensional analysis is performed to fit the proper fitted equation with the results; however, in reality drag reduction can be dominantly differed from the predictions due to the variations of operating conditions and flow geometry, so in this work it is attempted to provide a conceptual framework wherein the effects of operating conditions such as oil fraction, mixture velocity, density, viscosity, polymer concentration and polymer hydrodynamic volume on drag reduction be involved in the proposed model, since most widely used model in pipeline industry correlates drag reduction as a function of DRA concentration alone, [21]. The proposed model has been validated over a wide range of operating conditions, polymer concentrations for several types of commercially available polymer. The predicted model latter is validated and modified by the available software Design Expert to figure out the best format of the fitting equation.

\section{EXPERIMENTAL SETUP}

\section{A. Description of the Flow Loop}

A schematic process flow diagram of the rig used in this study is illustrated in Fig. 1. The experiments conducted in an in-house flow loop made of acrylic pipe with inner diameter (ID) of $30.6 \mathrm{~mm}$. The flow loop is made up of three sections namely storing section, regulating section and test section. The storing section consists of two $1.6 \mathrm{~m} 3$ storage tanks (water and oil) and separator tank of $2.0 \mathrm{~m} 3$ capacity. The mixture is delivered to the separator tank and separation process is enhanced with the help of a baffle plate inside the tank. A high speed camera (Fastec-Troubleshooter; FASTECIMAGING, USA) was used for visual observation of the flow patterns. After the flow section, the mixture was returned to a separator tank. Honeywell ST 3000 Smart differential pressure transmitter (which has a full scale accuracy of $0.0375 \%$ ) was connected to the pipe at $10 \mathrm{~m}$ from the inlet of the test section to measure the pressure drop through two pairs of pressure tapping over $1 \mathrm{~m}$ gap. The master polymer solution with a concentration of $1000 \mathrm{ppm}$ was injected to the water flow line before the oil-water mixing point using a MASTERFLEX pump (Master flex L/P digital console pump drive, manufactured in USA by Cole-Parmer $\left.{ }^{\circledR}\right)$. The experimental uncertainties of the pressure gradient (for all low $0.8 \& 1.0 \mathrm{~m} / \mathrm{s}$ and high velocity $1.2 \& 1.5 \mathrm{~m} / \mathrm{s}$ ) measurements were estimated to be within $\pm 8.0 \%$, and flowmeter measurements uncertainty were $\pm 2.0 \%$ and $\pm 3.0 \%$ for high and low velocities respectively.
The rig is equipped with a computer data acquisition system to automatically record the measured pressure drops. The drag reduction percentage (\%DR) was calculated based on the recorded pressure drop data through the test section using the following equation:

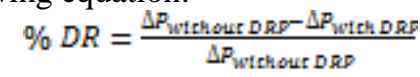

Where $\Delta P_{\text {without } D R P}$ and $\Delta P_{\text {with } D R P}$ are the pressure drop without and with DRP injection, respectively. This study was carried out in a horizontal pipe of 24-m long and 30.6-mm ID. The working fluids used in this study were tap water and hydraulic fluid based on mineral oil (Shell Tellus S2 V 15) and tap water, with viscosity of $24,1 \mathrm{cP}$ and density of 872,998 $\mathrm{kg} / \mathrm{m}^{3}$ respectively at $25^{\circ} \mathrm{C}$. The variables such as mixture velocity, input oil volume fraction and polymer concentration were considered in this study. The mixture velocities used were $0.8,1.0$ and $1.5 \mathrm{~m} / \mathrm{s}$, and for each of the mixture velocity, the flow rates of the oil and water were changed to give input oil volume fraction of $0.1,0.3$ and 0.5

\section{B. Polymer Preparation}

The high \& low molecular weight water-soluble anionic polymers used in this study, are manufactured by SNF Floerger, France with the commercial names of FLOPAAM3630, FLOPAAM3430S, AN934BPM, AN105SH, AN113SH, AN125, AN125SH and AN125VLM with charge density of 5 to $30 \%$. The polymers' properties are tabulated in Table I. A paddle mixer used to prepare a master solution of the polymer (mixture) solution with concentration of 1000 ppm, before the day of experiment commencement. A known mass of polymer powder was gently added to the shoulder of the vortex formed by the water. The stirring was allowed for 4 hours for the mixture to be completely homogenized after which the master solution was left overnight to ensure complete dissolution. The concentration of $1000 \mathrm{ppm}$ was chosen $(0.1 \% \mathrm{w} / \mathrm{w})$ to assures that the solution remains in the range of dilute solution to achieve homogeneous drag reduction where the polymer solution gets dissolved even at short distances after injection point, [22]

TABLE I POLYMERS' PROPERTIES AT $30^{\circ} \mathrm{C}$.

\begin{tabular}{|c|c|c|c|c|c|c|c|}
\hline 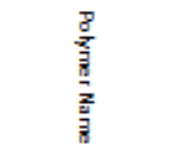 & 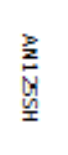 & $\frac{2}{2}$ & 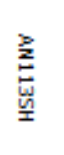 & 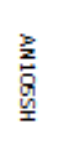 & 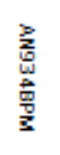 & 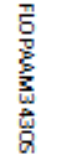 & 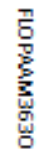 \\
\hline $\begin{array}{c}\text { Intrinsic Viscosity } \\
\qquad\left(\mathrm{cm}^{2} / \mathrm{g}\right)\end{array}$ & 7,320 & 6,359 & 6440 & 5058 & 4530 & 8024 & 8424 \\
\hline$M W(m D ;)$ & 12 & $6-8$ & 12 & 12 & $5-7$ & 12 & 20 \\
\hline $\begin{array}{c}\text { Charge Density } \\
\text { (\%) }\end{array}$ & 25 & 25 & 13 & 5 & 30 & 30 & 30 \\
\hline
\end{tabular}




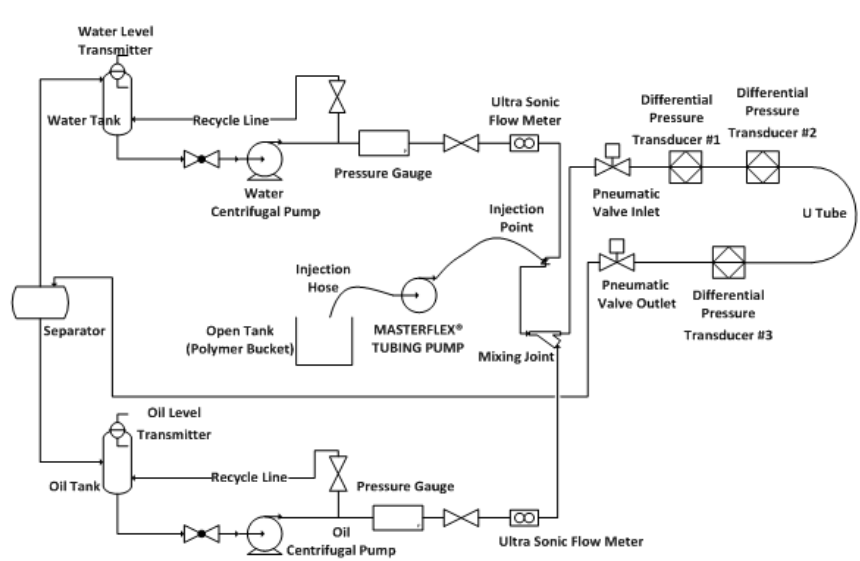

Fig. 1: Schematic of the multiphase flow loop

\section{RESULTS AND DISCUSSION}

\section{A. Flow Pattern}

Several flow patterns at different flow rates of oil/water mixtures and oil fraction as observed and reported by [23], was used. They are stratified (ST), dual continuous (DC), dispersed oil in water (Do/w) and dispersed water in oil (Dw/o) flow patterns. Since the focus of this study is the drag reduction in the dispersed phase region, mixture velocities used in this work were $0.8,1,1.2$ and $1.5 \mathrm{~m} / \mathrm{s}$ at oil fractions of $0.1,0.3,0.5$ and 0.7 for each of mixture velocity.

\section{B. Effect of Polymer Concentration}

Among the whole results of different polymers at different mixture velocities and input oil volume fractions, drag reduction increased with increase in the polymer concentration reaching plateau values effectively at polymer concentration of 30 ppm. Fig. 2 shows this dependency of drag reduction on the polymer concentration. In addition, there was a spike in the drag reductions up to concentration of $10 \mathrm{ppm}$, before the drag reductions reaching plateau values. This trend was also reported, [24], however it was found that the maximum drag reduction is around $20 \mathrm{ppm}$ polymer concentration. It should be noticed that the flow patterns remain unchanged after the polymer injection for the flow conditions used in this study. This is proven by visual observation in this study and quantitatively presented by [23]. Typical to previous drag reduction studies ([25], [26]), drag reduction increases with polymer concentration to plateau values above polymer concentration of $20 \mathrm{ppm}$.

\section{Effect of Oil Fraction}

Drag reduction decreases monotonically with the increase in oil fraction, showing lower drag reduction, Fig. 3 illustrates this effect when the maximum drag reduction ranging 13-37\% for oil fraction of 0.1 (Fig. 2) is decreased to range of 5-32\% at oil fraction of 0.3 at same velocity as illustrated in Fig. 3. However the drag reduction was not observed at higher oil fraction i.e. 0.7, which can be deduced that the water-soluble polymers are effective drag reducers when the water phase is dominant, [26].

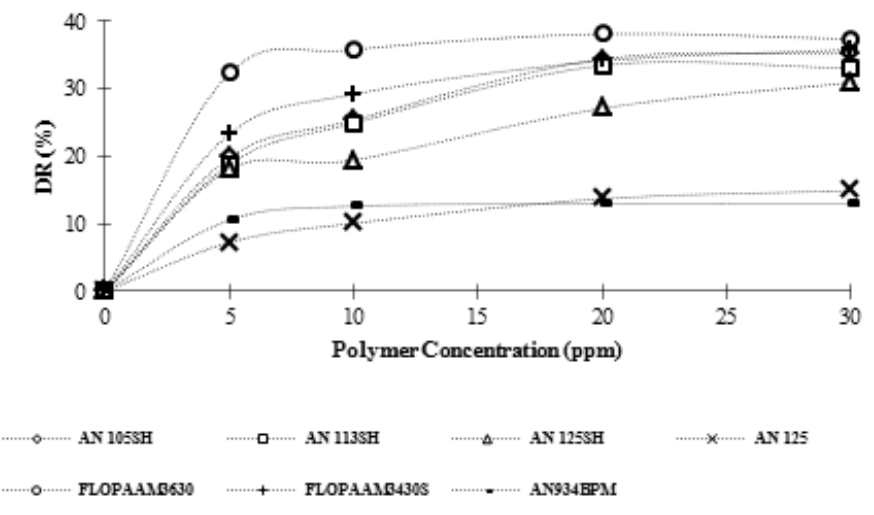

Fig. 2: Drag reduction at $0.8 \mathrm{~m} / \mathrm{s}$ and oil fraction of 0.1

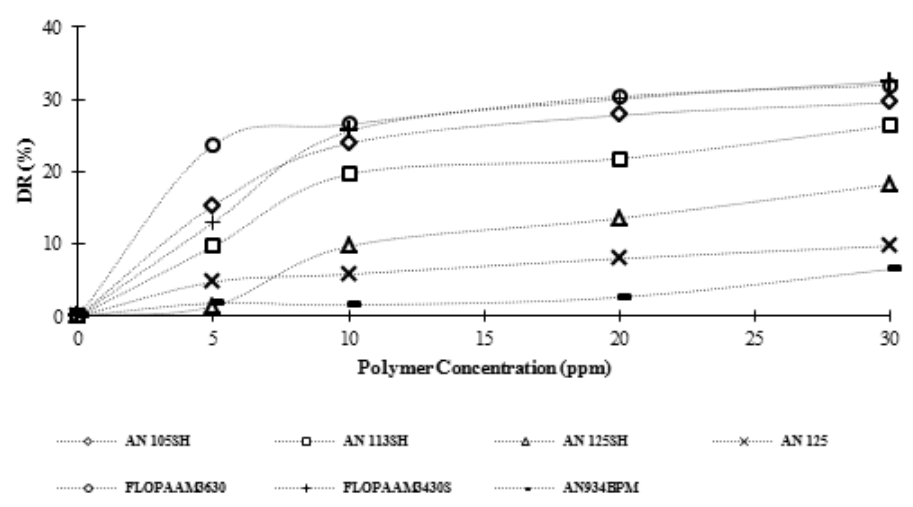

Fig. 3: Drag reduction at $0.8 \mathrm{~m} / \mathrm{s}$ and oil fraction of 0.3

In addition in the Fig. $2 \& 4$ the effect of mixture velocities of 0.8 and $1.5 \mathrm{~m} / \mathrm{s}$ is presented, when increasing the velocity leads in increase in drag reduction.

\section{Effect of Polymer Molecular Weight}

The effect of molecular weight on the performance of the DRP is illustrated in the Fig. 5 for three polymers FLOPAAM3630, FLOPAAM3430S and AN934BPM with molecular weight of 20,12 and 5-7 MDa. Drag reduction reached maximum values of around $45 \%$ at polymer concentration of $30 \mathrm{ppm}$ for the highest molecular weight polymer (i.e. FLOPAAM3630), and a maximum value of $20 \%$ is found using the low molecular weight AN934BPM. It can be observed that the increase in molecular weight increased the drag reduction as it was extensively reported ([27]; [28]; [7]; [26]; [29]).

\section{E. Effect of Charge Density}

In the previous study of the authors the effect of the charge density is widely investigated and turned out that polymers with lower charge density and same molecular weight resulted in higher drag reduction, interested readers may refer to the study by [30], since the molecular weight and the charge density have great effect on the hydrodynamic volume of the polymer chain in solution, which can be indicated by the intrinsic viscosity, so higher hydrodynamic volume results in higher intrinsic viscosity, [31]. 


\section{F. Model Development}

\section{Fuzzy Set Theory and Fuzzy Logic System}

As it is discussed in the text, the drag reduction depending on many factors which leads in more complexity of the phenomena. More complex a system, less ability to make precision and significant statements about the behavior, until reaching a limit beyond which precise and significance become almost mutually exclusive characteristics, [32]. Up to here five known different factors (inputs) are controlling the drag reduction in this study which are; mixture velocity, oil fraction, molecular weight, charge density and polymer concentration and only on output (i.e. DR\%) is unknown. In a real modeling it seems to be difficult to find out a model which fits this problem, so the fuzzy logic toolbox in Matlab is a proper option which can relate the five inputs to one output. For this purpose the different parts of the fuzzy set are determined as rule base, membership function. In the rule base, the linguistic description provided by the expert are introduced to define the rules. For example the term "charge density" is used to describe the linguistic variable charge density and the linguistic value of this variable take on the values of "high" and "low" or the variable "DR\%" takes values of "low", "medium" and "high". Note that we are using "medium" as an abbreviation for "medium drag reduction \%" and so on for the other variables. Describing the process with linguistics is not always easy, then better understanding of the process parameters and dynamic generally gives a better linguistic quantification. Next, we will use the above linguistic description to specify a set of rules (a rule-base) that obtain the expert's knowledge about how to control the plant.

Since the number of inputs are five, then for each one two linguistic value are defined which gives $25=32$ rules to describe the plant. For the current case, we have the following rules (no quotes shown, since the whole rule is linguistic):

If (Charge density is Low charge) and (Oil Fraction is Low oil) and (Mixture Velocity is Low velocity) and (Molecular Weight is High MW) and (Concentration is Low Concentration) then (Drag Reduction is Low DR)

If (Charge density is High charge) and (Oil Fraction is Low oil) and (Mixture Velocity is High velocity) and (Molecular Weight is High MW) and (Concentration is Low Concentration) then (Drag Reduction is High DR), etc.

Using the same approach, it is possible to write down rules for the drag reduction for all possible cases. Up to this point it is quantified the expert's knowledge how to control the process. Then the expert needs to quantify the meaning of the linguistic values using "membership functions."

The membership function quantifies, whether values of inputs (linguistic variables) are members of the set of values that are defined as "high" or "low". Depending on the application and the designer, there are different choices of membership functions are possible, like bell-shaped function, a trapezoid, or other asymmetrical functions, therefore the membership function associated with linguistic value and maps linguistic variable to $[0,1]$." Fuzzy logic then is used to heuristically quantify the meaning of linguistic variables, linguistic values, and linguistic rules.

The average percent error is defined as

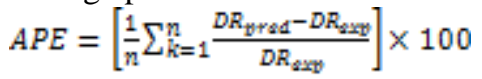

Where subscripts "pred" and "exp" represent the predicted and experimental values, respectively. The average absolute percent error (AAPE) is calculated to evaluate the prediction capability of the model by fuzzy logic, equation is given by

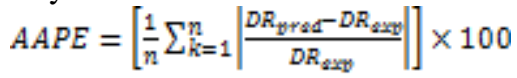

The standard deviation of the predicted value from the experimental is used to measure the spread of the data about the mean value. The equation which is also known as the root mean square percent error can be expressed as

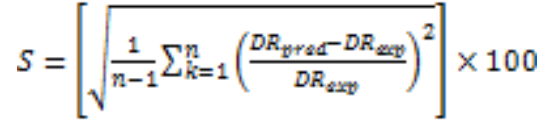

It should be noticed that due to negative values of some of the data, they are not used herein this section. Then for each oil fraction, 28 results (at 4 different polymer concentration and 7 polymer) were read which gives 336 individual results.

Graphical presentation of the fuzzy logic outputs are illustrated in the Fig. 6, which is demonstrating the DR\% vs MW and polymer charge density which implies that by increasing the molecular weight the DR\% as expected is increased. From Fig. 6 also it is observed that if increasing the charge density is in favor of the molecular weight increase, an advantage of drag reduction efficiency rise up is expectable, for instance following line $35 \%$ of drag reduction shows that a by increasing the charge density for a constant DR\% the molecular weight must be reduced, but this is up to median value of $20 \%$ after which the increase in molecular weight is inevitable, or it could be mentioned that the molecular weight has more pronounce effect as one of the limiting factor in the drag reduction phenomena.

The following Table II presents the actual and predicted values of DR\% at different conditions, alongside the average percent error, (APE), average absolute percent error (AAPE). The negative percent error shows that the values of predicted are less than the measured values, and this error is higher at all velocities around the oil fraction of 0.5 , however there is no good reason for this except the phase inversion phenomena which ordinarily happens around this fraction of oil when the dominant phase is changed and the pressure gradient is at its highest value. 
TABLE II

THE AAD\%, AAPE\% NAD STANDARD DEVIATION OF THE PREDICTED RESULTS OF DR\% BY FUZZY LOGIC

\begin{tabular}{|l|l|l|l|l|l|l|l|l|l|l|l|l|l|}
\hline Velocity & \multicolumn{1}{|l}{$\mathbf{0 . 8} \mathbf{~ m} / \mathbf{s}$} & $\mathbf{1 . 0} \mathbf{~ m} / \mathbf{s}$ & $\mathbf{0 . 2} \mathbf{~ m} / \mathbf{s}$ & \multicolumn{3}{l|}{$\mathbf{1 . 5} \mathbf{~ m} / \mathbf{s}$} \\
\hline Oil Fraction & $\mathbf{0 . 1}$ & $\mathbf{0 . 3}$ & $\mathbf{0 . 5}$ & $\mathbf{0 . 1}$ & $\mathbf{0 . 3}$ & $\mathbf{0 . 5}$ & $\mathbf{0 . 1}$ & $\mathbf{0 . 3}$ & $\mathbf{0 . 5}$ & $\mathbf{0 . 1}$ & $\mathbf{0 . 3}$ & $\mathbf{0 . 5}$ \\
\hline $\begin{array}{l}\text { APE (\%) } \\
\text { count=28 }\end{array}$ & -12.2 & -13.2 & -15.8 & -12.6 & -13.6 & -19.6 & -11.3 & -17.2 & -19.8 & -12.8 & -17.2 & -18.8 \\
\hline AAPE (\%) & 13.1 & 15.5 & 16.0 & 12.6 & 14.7 & 22.2 & 12.6 & 17.2 & 24.8 & 12.8 & 17.5 & 19.2 \\
\hline STDV (\%) & 13.7 & 13.8 & 15.5 & 6.9 & 10.8 & 23.8 & 12.8 & 28.1 & 14.2 & 9.6 & 13.6 & 11.6 \\
\hline
\end{tabular}

The values of the DR\% after phase inversion are negative that could be due to two reasons, first the nature of the polymer which is a water-soluble one, so in the circumstance of oil dominant phase the polymer is not applicable anymore, [16]; second the change in the flow property which is shifted from turbulent to a laminar flow where the drag reduction is not taking place in the laminar region, [33]. Therefore the prediction is not fairly accurate at this point.

\section{Predictive Model}

It has been revealed that concentration, hydrodynamic volume, density, flowrate, viscosity, oil fraction and flow geometry have significant effects on the drag reduction. Therefore, using Buckingham theorem for a total of seven variables (i.e. DR, density, viscosity, velocity, pipe diameter and dimensionless hydrodynamic volume and dimensionless concentration) with three repeating variables (i.e. density, viscosity and pipe diameter), the three terms become:

$\pi_{1}=$ Reynolds Number

$\pi_{2}=$ Dimensionless Concentration

$\pi_{3}=$ Dimensionless Hydrodynamic volume

$\pi_{4}=D R$

$$
\text { And } \pi_{4}=f\left(\pi_{1}, \pi_{2}, \pi_{3}\right)
$$

Where hydrodynamic volume is a characteristic of polymer obtained by multiplying of the concentration by its intrinsic viscosity.

The mixture density and viscosity in dispersed flow as shown in Eqs. 6 and 7, where $\varepsilon$ denotes the volume fraction of the dispersed phase and $\rho c$ and $\rho d$ denote the densities of the continuous and dispersed phases respectively.

$$
\rho_{m}=\rho_{c}(1-\varepsilon)+\rho_{d} \varepsilon
$$

Various models for the emulsion viscosity have been proposed in the literature [see e.g. [34], [35]] and in this study the model as proposed by [36] is used, in which the constants $\mathrm{c} 1$ and $\mathrm{c} 2$ are dependent on the continuous phase according to: $c 1=1.558$ and $c 2=1.66$ for oil continuous flow and $c 1=1.307$ and $\mathrm{c} 2=1.18$ for water-continuous flow.

$$
\mu_{m}=\mu_{c}\left(1+\frac{c_{1} \varepsilon}{c_{2}-c_{1} \varepsilon}\right)^{2.5}
$$

Different forms of functions were proposed for the best reduction in variability as a function of Reynolds number, hydrodynamic volume and polymer concentration. t However, the negative values of the DR for some of the polymers in the previous study by this group created aa major problem., Because of this, the drag reduction equation was reordered to give the pressure ratio as was shown earlier in the text, Therefore, the dimensionless pressure ratio term "PR" was used in the functions instead of the term 'DR' and the equation changed to the following form:

$$
D R=a \times R e^{n_{1}} \times p p m^{n_{2}} \times H V^{n_{\mathbb{I}}}
$$

Using the available method of error minimization (Least Squares Method) the above equation was fitted to the 384 results of different polymers in the rig and the following values were found

$$
\mathrm{a} 1=2.029, \mathrm{n} 1=-0.103, \mathrm{n} 2=-0.115, \mathrm{n} 3=-0.191
$$

The $\mathrm{R} 2$ value was 0.3501 , meaning that the proposed model is not very good for this study. To improve the model, its format was changed and coded value of the data was used in this stage instead of dealing with a large number of data which is cumbersome. Thereafter, the results are transformed to a domain of $[-1,1]$ using the following equation;;

$y_{\text {coded }}=1+2 \frac{y-y_{\max }}{y_{\max }-y_{\min }}, \quad-1 \leq y_{\text {coded }} \leq 1, \quad y_{\min } \leq y \leq y_{\max }$

Taking advantage of this method, three more different form of proposed model were fitted to investigate the best fitting equation, so the general form of the proposed equation will be as follows:

$$
\begin{gathered}
D R=a_{0}+a_{1} R e+a_{2} p p m+a_{3} H V+b_{1} R e \times p p m+b_{2} R e \times H V+b_{3} p p m \times H V+c_{1} R^{2}+c_{2} p p m^{2} \\
+c_{3} H V^{2}
\end{gathered}
$$

The possible equations are linear, 2 Factorial and Quadratic one. Using the same method of error minimization in Excel Table III gives the constants for each equation as well where it gives values of $R^{2}=0.4211, \quad R 2=0.4540$ and $R 2=0.6028$ for linear, 2Factorial and Quadratic respectively.

The impact of selected three parameters: was investigated on PR, by the response-surface methodology using design expert. So the experimental results were analyzed through RSM to obtain an empirical model for the best response. As it was expected the quadratic model was suggested to explain the mathematical relationship between the independent variables and dependent responses as it is represented by the former equation in the text. The calculated Fischer values (F-test) and thereby the probability values ( $P$ value) were calculated, and the significance of each parameter was identified for $P$ values of lower than 10\%, as can be seen in Table IV.

The Model F-value of 63.06 implies the model is significant. Values of "Prob > F" less than 0.05 indicate model 
terms are significant. In this case a1, a2, a3, b2, c1, c2 and c3 are significant model terms. Values greater than 0.1 indicate the model terms are not significant. The adjusted coefficient of determination was calculated sufficiently high $(\mathrm{R} 2$ adj $=$ 0.5932), which shows a good agreement between the model and experiments.

The residual analysis of the model is presented in Fig. $7 \&$ 8. Fig. 7 represents the normal probability plot of the residuals, which shows a linear relationship. It proves that the random error is independently and normally distributed.

TABLE III: CONSTANT OF THE PROPOSED MODEL

\begin{tabular}{|l|l|l|l|}
\hline Constants & Linear & 2Factorial & Quadratic \\
\hline $\mathbf{a}_{\mathbf{0}}$ & 0.7407 & 0.7416 & 0.7935 \\
& & & \\
\hline $\mathbf{a}_{\mathbf{1}}$ & -0.1219 & -0.1187 & -0.1236 \\
\hline $\mathbf{a}_{\mathbf{2}}$ & -0.0775 & -0.0731 & -0.0736 \\
\hline $\mathbf{a}_{\mathbf{3}}$ & -0.0637 & -0.0850 & -0.0806 \\
\hline $\mathbf{b}_{\mathbf{1}}$ & $\bullet$ & 0.0145 & 0.0145 \\
\hline $\mathbf{b}_{\mathbf{2}}$ & $\bullet$ & -0.0702 & -0.0702 \\
\hline $\mathbf{b}_{\mathbf{3}}$ & $\bullet$ & -0.0070 & -0.007 \\
\hline $\mathbf{c}_{\mathbf{1}}$ & $\bullet$ & $\bullet$ & -0.0293 \\
\hline $\mathbf{c}_{\mathbf{2}}$ & $\bullet$ & $\bullet$ & 0.0389 \\
\hline $\mathbf{c}_{\mathbf{3}}$ & $\bullet$ & $\bullet$ & -0.1483 \\
\hline
\end{tabular}

TABLE IV

EFFECTS AND MODEL COEFFICIENTS FOR PRESSURE RATIO

\begin{tabular}{|c|c|c|c|}
\hline & & & \\
& & & \\
\hline MoDEL & & 63.06375 & $<0.0001$ \\
\hline A0 & 0.79 & $\bullet$ & $\bullet$ \\
\hline A1 & -0.12 & 19.0041 & $<0.0001$ \\
\hline A2 & -0.074 & 94.45911 & $<0.0001$ \\
\hline A3 & -0.081 & 80.36858 & $<0.0001$ \\
\hline B1 & 0.014 & 1.709355 & 0.1919 \\
\hline B2 & -0.070 & 28.87724 & $<0.0001$ \\
\hline B3 & $-7.005 \mathrm{E}-3$ & 0.447043 & 0.5042 \\
\hline C1 & -0.029 & 4.043287 & 0.0451 \\
\hline C2 & 0.039 & 9.470177 & 0.0022 \\
\hline C3 & -0.15 & 126.5586 & $<0.0001$ \\
\hline
\end{tabular}

Fig. 8 presents predicted model versus experimental response that shows a negligible scattering pattern around the bisector, [37].This quantitative and qualitative approach indicates that constructed model can predict the experiments with fairly well precision.

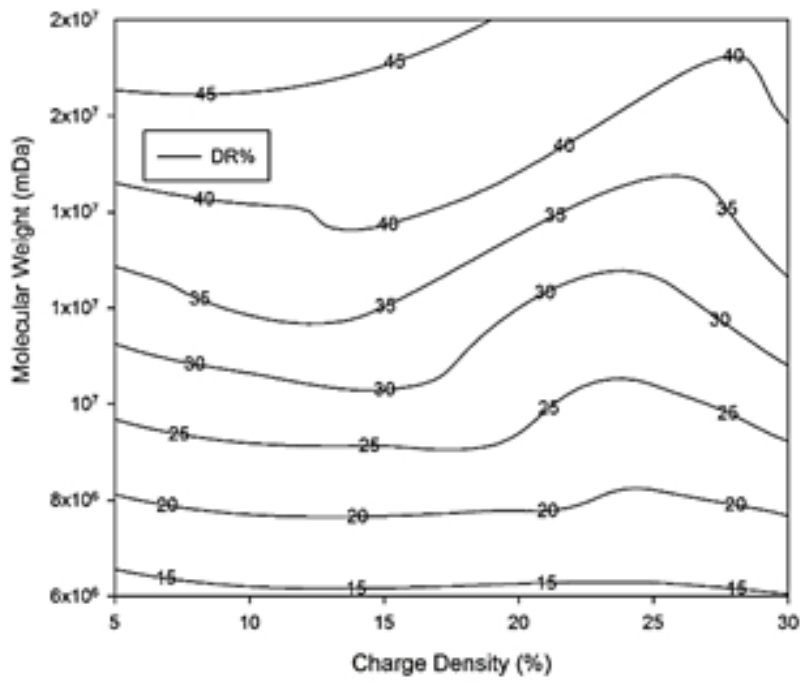

Fig. 6: Contour demonstration of DR\% variation at oil fraction of 0.1 at velocity of $1.0 \mathrm{~m} / \mathrm{s}$ vs. MW and Charge density

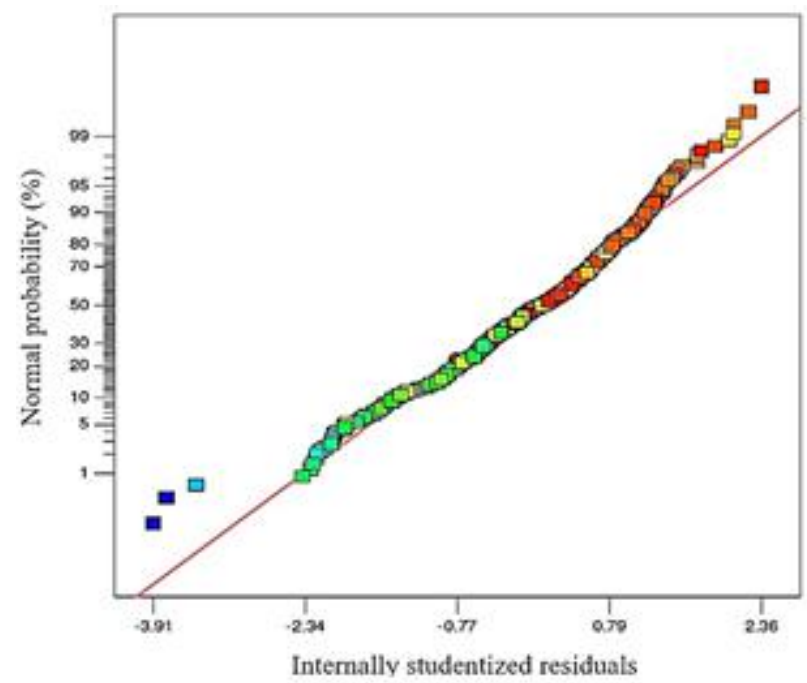

Fig. 7: Normal probability plot of residuals, Quadratic form

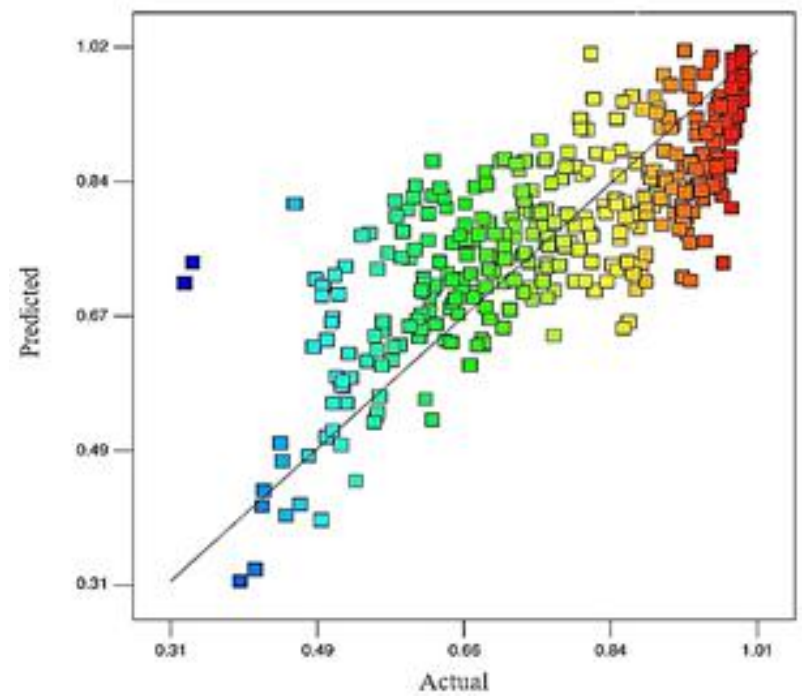

Fig. 8: predicted values against the actual values of response 


\section{CONCLUSION}

Drag reduction phenomena is an example of the complicated phenomena in the industry and literature where the phenomenon depends on many parameters such as molecular weight, hydrodynamic volume, polymer structure, and etc. In addition the flow geometry also affects the performance of DRPs as well, therefore its prediction needs a good knowledge of the condition that gains by the experts through the process. Fuzzy logic as a superset of standard logic deals with the partial truth, can be used to solve real life problems. In this study the performance of different DRPs whit different properties were investigated in a series of experiment and then predicted via fuzzy logic whit in a good agreement with the experimental results. Additionally the effort was done to fit different equations with the experimental results, where eventually a quadratic equation could hand in the best fitting for the experimental results. In addition the results implied that the effects of molecular parameters of the polymer involved in the chain flexibility in solution, are crucial in its ability to be an effective drag reducing agent in multiphase oil-water flow. For the investigated polymers in this study, polymer flexibility is enhanced with higher molecular weight and lower percentage of charged groups in the main backbone of the polymer chain. Only in the case of increasing the molecular weight the adverse effect of charge density increase is negligible

\section{ACKNOWLEDGEMENT}

The authors would like to thank The Research Council (TRC), Oman, for providing the funds for this research work and Sultan Qaboos University, Oman, for providing enabling environment to carry out the research.

\section{REFERENCES}

[1] Toms, B.A., Some observation on the flow linear polymer solution Trough straight tubes at large Reynolds number, in Proceeding of the First International Congress on Rheology.1948: North Holland, Amsterdam. p. 135-141.

[2] Elperin, I.T., B.M. Smolski, and S. Leventhal, Decreasing the Hydrodynamic Resistance of Pipelines. International Journal of Chemical Engineering 1967. 7: p. 276-295.

[3] Sylvester, N.D. and J.S. Tyler, Dilute Solution Drag-Reducing Properties of Polymers. Ind. Eng. Chem. Prod. Res. Develop, 1970. 9(4). http://dx.doi.org/10.1021/i360036a016

[4] Liaw, G.-C., J.L. Zakin, and G.K. Patterson, Effects of molecular characteristics of polymers on drag reduction. AIChE Journal, 1971. 17 (2): p. 391-397. http://dx.doi.org/10.1002/aic.690170228

[5] Mowla, D., M.S. Hatamipour, and M. Moshfeghian, The Effect of Dilute Polymer Solutions on Drag Reduction in Horizontal Two Phase Flow. Int. J. for Eng. Analysis \& Design, 1995. 2(A): p. 97-105.

[6] Burger, E.D., W.R. Munk, and H.A. Wahl, Flow increase in the transalaska pipeline through use of a polymeric drag-reducing additive. JPT, Journal of Petroleum Technology, 1982. 34(2): p. 377-386. http://dx.doi.org/10.2118/9419-PA

[7] Zakin, J.L. and D.L. Hunston, Effect of polymer molecular variables on drag reduction. Journal of Macromolecular Science, Part B, 1980. 18(4): p. 795-814. http://dx.doi.org/10.1080/00222348008241866

[8] Witold Brostow, J Hamide Ertepinar, and R.P. Singhi, Flow of Dilute Polymer Solutions: Chain Conformations and Degradation of Drag Reducers. Macromolecules 1990. 23: p. 5109-5118. http://dx.doi.org/10.1021/ma00226a013

[9] Schulz Donald, N., J.E. Glass, and C.F. Zukoski, Polymers as Rheology Modifiers, in Polymers as Rheology Modifiers. 1991, American Chemical Society. p. 2-17. http://dx.doi.org/10.1021/bk-1991-0462

[10] Mumick, P.S., et al., Water-Soluble Copolymers. 56. Structure and Solvation Effects of Polyampholytes in Drag Reduction. American Chemical Society, Macromolecules 1994. 27: p. 323-331. http://dx.doi.org/10.1021/ma00080a003

[11] Mumick, P.S., R.D. Hester, and C.L. McCormick, Water-Soluble Copolymers: 55. N-Isopropylacrylamide Copolymers in Drag Reduction: Effect of Molecular Structure, Hydration, and Flow Geometry on Drag Reduction Performance,. Polymer Engineering \& Science 1994. 34 p. 1429. http://dx.doi.org/10.1002/pen.760341810

[12] Abubakar, A., et al., Roles of drag reducing polymers in single- and multi-phase flows. Chemical Engineering Research and Design, 2013

[13] Virk, P.S., The Drag Reduction Fundamentals: A Brief Recapitulation, in Biotechnology of Marine Polysaccarides, Third Annual Lecture and Seminar Papers, E.R.P.a.A.J.S. R. COLWELL, Editor 1985, Hemisphere. Washington: Cambridge (USA). p. 149-193.

[14] Rochefort, S. and S. Middleman, Effect of molecular configuration of Xanthan gum drage reduction. AIP Conference Proceedings, 1986. 137(1): p. 117-127. http://dx.doi.org/10.1063/1.35522

[15] Singh, R.P., et al., The Effect of Grafted and Ungrafted Guargum on Turbulent Flow of Water and on Hydraulic Conductivity of Soil, in The Influence of Polymer Additives on Velocity and Temperature Fields, B. Gampert, Editor. 1985, Springer Berlin Heidelberg. p. 131-139. http://dx.doi.org/10.1007/978-3-642-82632-0_11

[16] Morgan, S.E. and C.L. McCormick, Water-Soluble copolymers XXXII: Macromolecular drag reduction. A review of predictive theories and the effects of Polymer structure. Progress in Polymer Science, 1990. 15(3): p. 507-549. http://dx.doi.org/10.1016/0079-6700(90)90005-L

[17] Dominguez, J.A. and C. Nieto, 'Fuzzy logic'system enhances pumping unit's engine operation. Oil and Gas Journal, 1998. 96(17).

[18] Inagaki, H., et al. Optimization of fuzzy membership functions using the random-search method and its application to direct digital control in pipelines. in The Sixth International Offshore and Polar Engineering Conference. 1996. International Society of Offshore and Polar Engineers.

[19] Zadeh, L.A., Fuzzy sets. Information and Control, 1965. 8(3): p. 338353. http://dx.doi.org/10.1016/S0019-9958(65)90241-X

[20] Zadeh, L.A., Fuzzy logic - a personal perspective. Fuzzy Sets and Systems, 2015. 281: p. 4-20. http://dx.doi.org/10.1016/j.fss.2015.05.009

[21] McAllister, E.W., 13 - Liquids-Hydraulics, in Pipeline Rules of Thumb Handbook (Sixth Edition), E.W. McAllister, Editor. 2005, Gulf Professional Publishing: Burlington. p. 359-415.

[22] Bewersdorff, H., et al., An investigation of possible mechanisms of heterogeneous drag reduction in pipe and channel flows. Rheologica acta, 1993. 32(2): p. 140-149. http://dx.doi.org/10.1007/BF00366677

[23] Abubakar, A., et al., Influence of drag-reducing polymer on flow patterns, drag reduction and slip velocity ratio of oil-water flow in horizontal pipe. International Journal of Multiphase Flow, 2015. 73(0): p. 1-10. http://dx.doi.org/10.1016/j.ijmultiphaseflow.2015.02.016

[24] Yusuf, N., et al., Experimental study on the effect of drag reducing polymer on flow patterns and drag reduction in a horizontal oil-water flow. International Journal of Heat and Fluid Flow, 2012. 37: p. 74-80. http://dx.doi.org/10.1016/j.ijheatfluidflow.2012.04.014

[25] Al-Wahaibi, T. and P. Angeli, Experimental studies on flow pattern transitions in horizontal oil-water flow. 6th International Conference on Multiphase Flow (ICMF 2007), Leipzig, Germany, 2007.

[26] Al-Yaari, M., et al., Effect of drag reducing polymers on oil-water flow in a horizontal pipe. International Journal of Multiphase Flow, 2009. 35(6): p. 516-524. http://dx.doi.org/10.1016/j.ijmultiphaseflow.2009.02.017 
[27] Virk, P.S., Drag reduction fundamentals. AIChE Journal, 1975. 21(4): p. 625-656.

http://dx.doi.org/10.1002/aic.690210402

[28] Verani, M., Effects of polymer concentration and molecular weight on the dynamics of visco-elasto-capillary breakup, in Dept. of Aeronautics and Astronautics.1977, Massachusetts Institute of Technology.

[29] Abubakar, A., et al., Parameters of Drag Reducing Polymers and Drag Reduction Performance in Single-Phase Water Flow. Advances in Mechanical Engineering, 2014. 2014: p. 8.

[30] Eshrati, M., et al., Drag reduction using high molecular weight polyacrylamides during multiphase flow of oil and water: A parametric study. Journal of Petroleum Science and Engineering, 2015. 135: p. 403-409.

http://dx.doi.org/10.1016/j.petrol.2015.09.028

[31] Sorbie, K.S., Polymer-improved oil recovery. 1991: Blackie. http://dx.doi.org/10.1007/978-94-011-3044-8

[32] Zadeh, L.A., Similarity relations and fuzzy orderings. Information sciences, 1971. 3(2): p. 177-200. http://dx.doi.org/10.1016/S0020-0255(71)80005-1

[33] Virk, P.S., et al., The Toms phenomenon: turbulent pipe flow of dilute polymer solutions. J. Fluid Mech., 1967. 30. http://dx.doi.org/10.1017/s0022112067001442

[34] Pal, R. and E. Rhodes, Viscosity/concentration relationships for emulsions. Journal of Rheology (1978-present), 1989. 33(7): p. 10211045 .

http://dx.doi.org/10.1122/1.550044

[35] Brinkman, H., The viscosity of concentrated suspensions and solutions. The Journal of Chemical Physics, 1952. 20(4): p. 571-571. http://dx.doi.org/10.1063/1.1700493

[36] Valle, A., Three phase Gas-Oil-Water Pipe Flow, 2000, Imperial College of Science, Technology and Medicine, London.

[37] Montgomery, D.C., Design and Analysis of Experiments, 8th Edition. 2012: John Wiley \& Sons, Incorporated 